\title{
On Quasi Multiplicative Function
}

\author{
Azizul Hoque*, Himashree Kalita* \\ Department of Mathematics, Gauhati University, Guwahati, India \\ *Corresponding author: ahoque.ms@gmail.com, himashree.kalita28@gmail.com
}

Received March 01, 2015; Revised April 07, 2015; Accepted June 02, 2015

\begin{abstract}
In this paper we introduce two new Arithmetic functions, that is, Quasi-Multiplicative (QM) and omega $(\omega)$ functions. The Omega $(\omega)$ function is based on Euler's Phi $(\phi)$ function and is used to find the sum of coprime integers. Euler's Phi $(\phi)$ function, Dedekind's psi $(\psi)$ function, the sigma $(\sigma)$ function and $\tau$-function play significant role in this work.
\end{abstract}

Keywords: arithmetic function, quasi-multiplicative function, omega function

Cite This Article: Azizul Hoque, and Himashree Kalita, "On Quasi Multiplicative Function." Turkish Journal of Analysis and Number Theory, vol. 3, no. 2 (2015): 68-69. doi: 10.12691/tjant-3-2-6.

\section{Introduction}

Recall that an Arithmetic function $[2,6] f$ is Multiplicative if for each pair of coprime integers $m$ and $n$, it is satisfied $f(m n)=f(m) f(n)$. Missana [5] established some significant results on multiplicative functions. Dehaye [3] constructed some algebraic structures using arithmetic functions. Some classical examples of multiplicative functions that have important meaning in Number theory are Euler's Phi $(\phi)$ function, Dedekind's psi $(\psi)$ function, the sigma $(\sigma)$ function and $\tau-$ function. Recently Hoque and Kalita [4] studied perfect numbers and their generalizations using these multiplicative functions. For any Positive integer $n>1$ having the factorization $n=p_{1}^{r_{1}} p_{2}^{r_{2}} \ldots p_{k}^{r_{k}}$, where $p_{1}, p_{2}, \ldots, p_{k}$ are distinct prime numbers and $k, r_{1}, r_{2}, \ldots, r_{k} \geq 1$ are integers, these functions admit the following multiplicative representations:

$$
\begin{aligned}
& \phi(n)=n \prod_{i=1}^{k}\left(1-\frac{1}{p_{i}}\right) \\
& \psi(n)=n \prod_{i=1}^{k}\left(1+\frac{1}{p_{i}}\right) \\
& \sigma(n)=\prod_{i=1}^{k}\left(\frac{p_{i}^{k_{i}+1}-1}{p_{i}-1}\right) \\
& \tau(n)=\prod_{i=1}^{k}\left(k_{i}+1\right) .
\end{aligned}
$$

In this paper, we introduce the notions of Omega $(\omega)$ function and quasi-multiplicative function. The Omega $(\omega)$ function is based on Euler's Phi $(\phi)$ function and is used to find the sum of coprime integers. We establish some important results on these two functions via Euler's Phi $(\phi)$ function, Dedekind's psi $(\psi)$ function, the sigma $(\sigma)$ function and $\tau$-function.

\section{Main Results}

Definition 2.1: An Arithmetic function $f$ is QuasiMultiplicative (QM) if for each pair of coprime integers $m$ and $n$ there exists a positive integer $\mathrm{k}$ such that $f(m n)=k f(m) f(n)$. The positive $k$ is defined as the multiplicative index of $f$.

It is clear that a quasi-multiplicative function with index one is Multiplicative.

Definition 2.2: For any positive integer $n$, we define the Omega function, $\omega(n)$ as the sum of the positive integers less than $n$ and relatively prime to $n$.

By Theorem 7.7 of [2], we see that $\omega(n)=\frac{1}{2} n \phi(n)$.

Proposition 2.1: If $f$ is a multiplicative function and $k$ is a positive integer then $g=\frac{1}{k} f$ is quasi-multiplicative function with index $k$.

Proof: Let $m$ and $n$ be any two positive and coprime integers. Then

$$
\begin{aligned}
& g(m n)=\left(\frac{1}{k} f\right)(m n)=\frac{1}{k} f(m n) \\
& =\frac{1}{k} f(m) f(n)=k g(m) g(n) .
\end{aligned}
$$

This proves the result.

Theorem 2.2: The function $\omega$ is quasi multiplicative with index 2.

Proof: By definition,

$$
\omega(n)=\frac{1}{2} n \phi(n) .
$$


Since $\phi$ is multiplicative, the function $f$ defined by $f(n)=n \phi(n)$ is also multiplicative.

Thus by Proposition 2.1, $\omega$ is quasi multiplicative with index 2.

Proposition 2.3: For any prime number $p$,

$$
2 \omega(p)=(\sigma(p)-1)(\sigma(p)-2) .
$$

Proof: We have

$$
\omega(p)=\frac{1}{2} p \phi(p)=\frac{1}{2} p(p-1)
$$

Again

$$
\sigma(p)=p+1
$$

From equation (1) and equation (2), the result follows. Lemma 2.4 [1]: For every natural number $n \geq 2$,

$$
\phi(n) \psi(n) \sigma(n) \geq n^{3}+n^{2}-n-1
$$

Proposition 2.5: For every natural number $n \geq 2$,

$$
2 \omega(n) \psi(n) \sigma(n) \geq n^{4}+n^{3}-n^{2}-n .
$$

Theorem 2.6: There are infinitely many positive integers $m, n$ and $t$ such that

(i) $\phi(\omega(m))>\omega(\phi(m))>m$

(ii) $\omega(\phi(n))<\phi(\omega(n))<n^{2}$

(iii) $\omega(\phi(t))=2 \phi(\omega(t))$

Proof. (i) Let us suppose, $m=3.2^{k}$ for any positive integer $k$. Then

$$
\phi(m)=2^{k} \text { and } \omega(m)=3.2^{2 k-1} .
$$

Now, $\phi(\omega(m))=\phi\left(3.2^{2 k-1}\right)=2^{2 k-1}$

Also, $\omega(\phi(m))=\omega\left(2^{k}\right)=2^{2 k-2}=\frac{m 2^{k}}{6}>m$ for $k>3$.

Thus $\phi(\omega(m))>\omega(\phi(m))$ for $k \geq 1$.

Moreover, $\phi(\omega(m))>\omega(\phi(m))>m$ for $k>3$.

(ii) Let $n=2^{k} 5^{l}$ for any positive integer $k$ and $l$. Then

$$
\begin{aligned}
& \phi(n)=2^{k+1} 5^{l-1} \\
& \omega(n)=2^{2 k} 5^{2 l-1}
\end{aligned}
$$

Now, $\phi(\omega(n))=\phi\left(2^{2 k} 5^{2 l-1}\right)=2^{2 k+1} 5^{2 l-2}=\frac{2 n^{2}}{25}<n^{2}$.

Also $\omega(\phi(n))=\omega\left(2^{k+1} 5^{l-1}\right)=2^{2 k+2} 5^{2 l-3}=\frac{4 n^{2}}{125}$.

(iii) Let $t=2^{k}$ for any positive integer $k$. Then

$$
\begin{aligned}
& \phi(\omega(t))=2^{2 k-3}=\frac{t^{2}}{8} \\
& \omega(\phi(t))=2^{2 k-4}=\frac{t^{2}}{16} .
\end{aligned}
$$

Thus $\omega(\phi(t))=2 \phi(\omega(t))$.
Theorem 2.7: There are infinitely many positive integers $m, n$ and $t$ such that

(i) $m^{2}>\omega(\psi(m))>\psi(\omega(m))>m$

(ii) $n<\omega(\psi(n))<\psi(\omega(n))<n^{2}$

(iii) $\omega(\psi(t))=\psi(\omega(t))$

Proof. (i) Let $m=2^{k} 3^{l}$ for any positive integers $k$ and $l$. Then

$$
\begin{aligned}
& \psi(m)=2^{k+1} 3^{l} \\
& \omega(m)=2^{2 k-1} 3^{2 l-1}
\end{aligned}
$$

Now, $\omega(\psi(m))=\omega\left(2^{k+1} 3^{l}\right)=2^{2 k+1} 3^{2 l-1}=\frac{2 m^{2}}{3}<m^{2}$.

And, $\quad \psi(\omega(m))=\psi\left(2^{2 k-1} 3^{2 l-1}\right)=2^{2 k} 3^{2 l-1}>m$ for $l \geq 1$.

Thus we have $m^{2}>\omega(\psi(m))=2 \psi(\omega(m))>\psi(\omega(m))>m$.

(ii) Let $n=2^{k} 5^{l}$ for any positive integer $k$ and $l \geq 2$. Then

$$
\begin{aligned}
& \psi(n)=3^{2} 2^{k} 5^{l-1} \\
& \omega(n)=2^{2 k} 5^{2 l-1}
\end{aligned}
$$

Now, $\quad \omega(\psi(n))=\omega\left(3^{2} 2^{k} 5^{l-1}\right)=3^{3} 2^{2 k} 5^{2 l-3}=\frac{27 n^{2}}{125}>n$ for $l \geq 2$

And, $\psi(\omega(n))=\psi\left(2^{2 k} 5^{2 l-1}\right)=3^{2} 2^{2 k} 5^{2 l-2}=\frac{9 n^{2}}{25}<n^{2}$.

Thus we have $n<\omega(\psi(n))=\frac{3}{5} \psi(\omega(n))<\psi(\omega(n))<n^{2}$.

(iv) Let $t=2^{k}$ for any positive integer $k$. Then

$$
\begin{aligned}
& \psi(t)=2^{k-1} 3 \\
& \omega(t)=2^{2 k-2}
\end{aligned}
$$

Now, $\omega(\psi(t))=2^{2 k-3} 3$ and $\psi(\omega(t))=2^{2 k-3} 3$.

\section{Acknowledgements}

First author acknowledges UGC for Junior Research Fellowship (No.GU/UGC/VI(3)/JRF/ 2012/2985).

\section{References}

[1] Atanassov, K., Notes on $\phi, \psi$ and $\sigma$-functions. Part 6, Notes on Number Theory and Discrete Mathematics, Vol. 19, 2013, No. 1, 22-24.

[2] Burton, D. M., Elementary Number Theory, $6^{\text {th }}$ edi., Tata McGraw-Hill Pub.Com. Ltd, New Delhi.

[3] Dehaye, P. O., On the structure of the group of multiplicative Arithmetic functions, Bull. Belg. Math. Soc., 9, 15-21, 2002.

[4] Hoque, A. and Kalita, H., Generalised perfect numbers connected with Arithmetic functions, Math. Sci. Lett., 3(3), 249-253, 2014.

[5] Missana, M. V., Some results on multiplicative functions, Notes on Number Theory and Discrete Mathematics, 16 (4), 22-24, 2010.

[6] Sivaramakrishnan, R., Classical Theory of Arithmetic Function, New York, Dekker, 1989. 